\title{
Adjunct low-dose ketamine infusion vs standard of care in mechanically ventilated critically ill patients at a Tertiary Saudi Hospital (ATTAINMENT Trial): study protocol for a randomized, prospective, pilot, feasibility trial
}

Mohammed Bawazeer ${ }^{1^{*}+}$ (D) Marwa Amer ${ }^{2^{*}+}$, Khalid Maghrabi ${ }^{1}$, Kamel Alshaikh¹, Rashid Amin², Muhammad Rizwan ${ }^{1}$, Mohammad Shaban ${ }^{1}$, Edward De Vol ${ }^{3}$ and Mohammed Hijazi ${ }^{1}$

\begin{abstract}
Background: A noticeable interest in ketamine infusion for sedation management has developed among critical care physicians for critically ill patients. The 2018 Pain, Agitation/sedation, Delirium, Immobility, and Sleep disruption guideline suggested low-dose ketamine infusion as an adjunct to opioid therapy to reduce opioid requirements in post-surgical patients in the intensive care unit (ICU). This was, however, rated as conditional due to the very low quality of evidence. Ketamine has favorable characteristics, making it an especially viable alternative for patients with respiratory and hemodynamic instability. The Analgo-sedative adjuncT keTAmine Infusion iN Mechanically vENTilated ICU patients (ATTAINMENT) trial aims to assess the effect and safety of adjunct low-dose continuous infusion of ketamine as an analgo-sedative compared to standard of care in critically ill patients on mechanical ventilation (MV) for $\geq 24 \mathrm{~h}$.

\footnotetext{
* Correspondence: mbawazeer@kfshrc.edu.sa; mamer@kfshrc.edu.sa

${ }^{\dagger}$ Mohammed Bawazeer and Marwa Amer contributed equally to this work.

'Department of Critical Care Medicine (MBC 94), King Faisal Specialist Hospital and Research Center, P.O Box 3354, Riyadh 11211, Saudi Arabia

${ }^{2}$ Pharmaceutical Care Division (MBC 11), King Faisal Specialist Hospital and Research Center, P.O Box 3354, Riyadh 11211, Saudi Arabia

Full list of author information is available at the end of the article
}

(c) The Author(s). 2020 Open Access This article is licensed under a Creative Commons Attribution 4.0 International License, which permits use, sharing, adaptation, distribution and reproduction in any medium or format, as long as you give appropriate credit to the original author(s) and the source, provide a link to the Creative Commons licence, and indicate if changes were made. The images or other third party material in this article are included in the article's Creative Commons licence, unless indicated otherwise in a credit line to the material. If material is not included in the article's Creative Commons licence and your intended use is not permitted by statutory regulation or exceeds the permitted use, you will need to obtain permission directly from the copyright holder. To view a copy of this licence, visit http://creativecommons.org/licenses/by/4.0/ The Creative Commons Public Domain Dedication waiver (http://creativecommons.org/publicdomain/zero/1.0/) applies to the data made available in this article, unless otherwise stated in a credit line to the data. 
(Continued from previous page)

Methods/design: This trial is a prospective, randomized, active controlled, open-label, pilot, feasibility study of adult ICU patients (> 14 years old) on MV. The study will take place in the adult ICUs in the King Faisal Specialist Hospital and Research Center (KFSH\&RC), Riyadh, Saudi Arabia, and will enroll 80 patients. Patients will be randomized postintubation into two groups: the intervention group will receive an adjunct low-dose continuous infusion of ketamine plus standard of care. Ketamine will be administered over a period of $48 \mathrm{~h}$ at a fixed infusion rate of $2 \mu \mathrm{g} /$ $\mathrm{kg} / \mathrm{min}(0.12 \mathrm{mg} / \mathrm{kg} / \mathrm{h})$ in the first $24 \mathrm{~h}$ followed by $1 \mu \mathrm{g} / \mathrm{kg} / \mathrm{min}(0.06 \mathrm{mg} / \mathrm{kg} / \mathrm{h})$ in the second $24 \mathrm{~h}$. The control group will receive standard of care in the ICU (propofol and/or fentanyl and/or midazolam) according to the KFSH\&RC sedation and analgesia protocol as clinically appropriate. The primary outcome is MV duration until ICU discharge, death, extubation, or 28 days post-randomization, whichever comes first.

Discussion: The first patient was enrolled on 1 September 2019. As of 10 October 2019, a total of 16 patients had been enrolled. We expect to complete the recruitment by 31 December 2020. The findings of this pilot trial will likely justify further investigation for the role of adjunct low-dose ketamine infusion as an analgo-sedative agent in a larger, multicenter, randomized controlled trial.

Trial registration: ClinicalTrials.gov: NCT04075006. Registered on 30 August 2019. Current controlled trials: ISRCTN14730035. Registered on 3 February 2020.

Keywords: Ketamine, Standard of care, Sedation, Critically ill, Mechanical ventilation, ATTAINMENT, Propofol, Fentanyl, Midazolam, Delirium, Vasopressors

\section{Background}

Sedation and analgesia management are both integral components of care in the intensive care unit (ICU). Although benzodiazepines have been the mainstay therapy for sedation in critically ill patients, their use has declined in recent years, with favoring of nonbenzodiazepines, such as propofol and dexmedetomidine. This change in practice is based on studies demonstrating the association between the sustained use of benzodiazepines and increased mechanical ventilation (MV) duration, ICU length of stay (LOS), and development of delirium. A paradigm shift has therefore occurred in the management of patients' sedation in the ICU. Maintenance of light levels of sedation in adult patients in the ICU has been recommended to improve patient clinical outcomes, such as shorter duration of MV and shorter ICU LOS [1].

A noticeable interest in ketamine infusion for sedation management in critically ill patients has developed among critical care physicians [2]. The 2018 Pain, Agitation/sedation, Delirium, Immobility, and Sleep disruption (PADIS) guideline suggested low-dose ketamine as an adjunct to opioid therapy for reducing opioid consumption in post-surgical adults admitted to the ICU (i.e., conditional recommendation, very low quality of evidence) [1]. In a single-center, double-blind, randomized controlled trial (RCT) of 93 ICU post-abdominal surgery patients, adjunctive ketamine was associated with a reduced intake of morphine. However, there were no differences in patients' self-reported pain intensity [3]. Ketamine was administered as $0.5 \mathrm{mg} / \mathrm{kg}$ intravenous (IV) push followed by infusion of $2 \mu \mathrm{g} / \mathrm{kg} / \mathrm{min}(0.12 \mathrm{mg} / \mathrm{kg} / \mathrm{h}) \times 24 \mathrm{~h}$, then $1 \mu \mathrm{g} / \mathrm{kg} /$ $\mathrm{min} \times 24 \mathrm{~h}(0.06 \mathrm{mg} / \mathrm{kg} / \mathrm{h})$. The incidence of side effects (i.e., nausea, delirium, hallucination, hypoventilation, pruritus, and sedation) did not differ between the ketamine and opioidalone groups. Based on this generally positive ICU RCT, the 2018 PADIS panel made a conditional recommendation for the use of low-dose ketamine as an adjunct to opioids to optimize acute post-operative pain management in critically ill adults (refer to Supplementary Table 1: Previous ketamine trials in the ICU setting) [2-11].

Because of an increased focus on ensuring that pain is appropriately controlled in patients before using sedative-hypnotic medications (also known as the analgo-sedation approach), ketamine has gained attention for its unique pharmacologic properties that could address both the analgesic and sedative requirements. Ketamine could result in decreased duration of MV while providing optimal levels of sedation [12]. Similar to dexmedetomidine, ketamine has a non-GABAergic mechanism of action [13]. It induces rapid sedation and analgesia through dual mechanisms mediated by inhibition of the $N$-methyl-D-aspartate receptor and activation of the opioid $\mu$ - and $\kappa$-receptors [14]. Ketamine is also Saudi Food and Drug Authority (FDA)-approved for the induction of anesthesia and has been used for acute and chronic pain in sub-anesthetic dose, post-operative opioid sparing, rapid sequence intubation, and procedural sedation and analgesia [15]. Additionally, ketamine has favorable characteristics, including bronchodilation, preservation of cardiac output, increase in blood pressure, minimal effects on bowel motility, and maintaining of respiratory drive and airway reflexes while actively weaning from MV; these features make it an especially viable alternative for patients with respiratory and hemodynamic instability [12]. 
Although commonly used sedatives are effective, they have side effects including benzodiazepine-associated delirium, opioid-induced constipation, and the negative hemodynamic effect caused by propofol and dexmedetomidine $[1,16-18]$. The most frequently observed adverse effects associated with ketamine when used to maintain sedation include tachycardia (6.7\%), hypertension (6\%), paradoxical agitation (up to $20 \%$ ), and hypersalivation (12\%) [12]. Although there is limited literature on adults, as many as $57 \%$ of pediatric patients who receive ketamine for continuous sedation experience the emergence phenomenon, including vivid hallucinations and delirium during or after ketamine use [19]. When ketamine is used for procedural sedation in adults (usually administered as a relatively high dose, $1-2 \mathrm{mg} / \mathrm{kg}$ repeated q5$15 \mathrm{~min}$ to maximum $100 \mathrm{mg}$ ), up to $20 \%$ of patients may develop the emergence phenomenon [20]. Risk factors for delirium with ketamine include prior history of psychiatric disorders, dementia, and the use of a high dose in procedural sedation [20]. The development of the emergence phenomenon can cause patients to transiently require higher amounts of other sedatives, usually benzodiazepines. However, ketamine-based analgo-sedation in MV patients administered as a subanesthetic/sub-dissociative/low dose results in similar numbers of delirium- and coma-free days as those in non-ketamine-based regimens, as shown in a retrospective cohort study conducted by Shurtleff et al. at an academic medical center [8]. Ketamine infusion in this trial was $5 \mu \mathrm{g} /$ $\mathrm{kg} / \mathrm{min}(0.3 \mathrm{mg} / \mathrm{kg} / \mathrm{h})$ titrated using $5 \mu \mathrm{g} / \mathrm{kg} / \mathrm{min}$ every 5 min up to a maximum of $25 \mu \mathrm{g} / \mathrm{kg} / \mathrm{min}(1.5 \mathrm{mg} / \mathrm{kg} / \mathrm{h})$. The authors found that the number of days alive without delirium or coma was 6 days (interquartile range [IQR] 2-9 days) with ketamine and 4 days (IQR 3-7 days) with a non-ketamine medication $(P=0.351)$. Delirium occurred in 29 of the 39 patients $(74 \%)$ with ketamine and in 34 of the 40 patients $(85 \%)$ with the non-ketamine drug $(P=0.274)$. Similarly, the RCT cited by the 2018 PADIS guideline showed that the incidence of side effects (i.e., delirium and hallucinations) did not differ between the ketamine and opioid-alone groups [3].

At King Faisal Specialist Hospital and Research Center (KFSH\&RC), continuous infusions of sedatives and analgesics are prescribed at the physician's discretion and titrated to achieve Richmond Agitation-Sedation Scale (RASS) and pain scores; the infusions are performed with a nurse-driven protocol. The protocol promotes analgesia-first sedation (with fentanyl) and recommends propofol as the first-line agent when sedation is required. Patients receive a daily spontaneous awakening trial (SAT) paired with a spontaneous breathing trial (SBT). The RASS and the Confusion Assessment Method for the ICU (CAM-ICU) are routinely used to assess the level of sedation and the presence of delirium, respectively. Ketamine, registered by the Ministry of
Health of Saudi Arabia, is a KFSH\&RC hospital formulary medication and is listed in the KFSH\&RC ICU pain and sedation protocol as an option for patients with severe bronchospasm. However, the order or the combination that could be most effective with ketamine is unclear (refer to Supplementary Figure 1: KFSH\&RC new sedation protocol for adult ICUs).

As stated previously, the 2018 PADIS guideline listed ketamine as a conditional recommendation, with very low quality of evidence (limited high-level evidence). Most trials listed in Supplementary Table 1 are in surgical ICU settings, retrospective in nature, or are RCTs focused on comparing ketamine to placebo or two study drugs (e.g., ketamine vs opioid). However, most patients in the ICU are sedated with a combination of drugs. Moreover, most trials had a limited focus on patientcentered outcomes, such as duration of MV or ICU LOS, as the primary outcome favoring surrogate outcomes, such as sedation scores and changes in analgesics and sedatives [2-11]. To help further delineate ketamine's role as a maintenance analgo-sedation agent in the ICU, further RCTs need to be conducted to compare the effects of ketamine to those of other analgesics and sedatives on reducing the duration of MV, ICU LOS, and delirium occurrence. Recently, there was a prospective, double-blinded, multicenter RCT (KeMiMof) in critically ill patients $>12$ years old and requiring sedation for $>24 \mathrm{~h}$ in the ICU, in Uganda. Patients were randomized to receive either ketamine-midazolam or morphinemidazolam given as premixed $50-\mathrm{ml}$ syringes for infusion. The primary outcome measures were duration of $\mathrm{MV}$, incidence of hypotension, and incidence of delirium. The trial was terminated on 28 August 2019 with pending results. Limitations of this trial are the use of premixed syringes, which are not typically used in adult ICU sedation practice, and the focus on comparing two study drugs (ketamine vs morphine) [21].

Robust clinical outcome data and comprehensive assessments of adverse events (AEs) associated with ketamine use in mechanically ventilated patients are limited, leaving a significant knowledge gap, which has been reflected in the wide variation in the use of ketamine as a sedative agent in ICUs. This is also highlighted in a recent systematic review and meta-analysis by Manasco et al. [22]. Therefore, we propose a prospective, randomized, active controlled, open-label, pilot, feasibility study to assess the effect and safety of Analgo-sedative adjunc $T$ keTAmine Infusion iN Mechanically vENTilated ICU patients (the ATTAINMENT trial) compared to standard of care alone. We hypothesized that low-dose ketamine infusion will reduce the duration of MV with an acceptable safety profile compared to standard of care. The findings of this pilot trial will likely justify further investigations on the role of adjunct low-dose 
ketamine infusion as an analgo-sedative and inform the design of a large multicenter RCT with sufficient power to detect differences in clinical outcomes.

\section{Study objectives \\ The primary objective}

The primary objective is to study the feasibility and effect of adjunct low-dose ketamine infusion on MV duration compared to the standard of care alone in critically ill patients.

\section{The secondary objectives}

Secondary objectives are to study the effect of adjunct low-dose ketamine infusion on the following:

1. The cumulative dose of pain and sedative medications

2. The incidence of dexmedetomidine use postrandomization

3. The number of patients within RASS and pain score goals

4. The hemodynamic status in terms of vasopressor therapy requirement

5. ICU and hospital LOS

6. Tracheostomy, unplanned extubation, and reintubation rates

7. The incidence of delirium and rate of positive CAM-ICU assessment

8. The rate of antipsychotic use for ICU-acquired delirium

9. The rate of hypersalivation and frequent suctioning

10. The rate of using physical restraint

11. Mortality rate at 28 days.

\section{Methods}

\section{Design overview}

The ATTAINMENT trial is a prospective, randomized, open-label, active controlled, parallel group, pilot, feasibility, phase 3 study of adult patients admitted to the KFSH\&RC adult ICUs, Riyadh, Saudi Arabia. This trial is approved by the Institutional Review Board (IRB) of the KFSH\&RC. The trial is registered in ClinicalTrials.gov: NCT04075006, current controlled trials: ISRCTN14730035, and Saudi Food and Drug Authority: SCTR \#19063002. The protocol adheres to the Standard Protocol Items: Recommendations for Interventional Trials (SPIRIT) guidelines (see Supplementary File 1 and Supplementary Table 2).

\section{Eligibility and enrollment}

The inclusion criteria are as follows:

- Adult patients ( $>14$ years old) on MV admitted to one of the following ICUs: medical, surgical, or transplant/oncology ICU

- Intubated within the previous $24 \mathrm{~h}$

- Expected to require MV for more than $24 \mathrm{~h}$
- Expected to be on the KFSH\&RC ICU sedation and pain protocol

- No objection from the ICU attending physician for enrollment

The exclusion criteria are:

- Patients with a history of dementia or psychiatric disorders or those on any antipsychotic or antidepressant medications at home

- Pregnancy

- Age $<14$ years old

- Expected to need MV $<24 \mathrm{~h}$

- Known hypersensitivity to ketamine

- Patients with expected targeted RASS score of -5 , e.g., patients on continuous infusion neuromuscular blockade

- Patients on dexmedetomidine as the primary sedative prior to randomization

- Patients with cardiogenic shock, acute decompensated heart failure, or myocardial infarction

- History of end-stage liver failure (Child-Pugh score C)

- Proven or suspected primary neurological injury (traumatic brain injury, ischemic stroke, intracranial hemorrhage, spinal cord injury, anoxic brain injury, brain edema)

- Patients with persistent heart rate $(\mathrm{HR})>150$ beats per minute (bpm) or systolic blood pressure (SBP) > $180 \mathrm{mmHg}$

- Patients identified as Do Not Resuscitate (DNR) or those expected to die within $24 \mathrm{~h}$

- Patients on extracorporeal membrane oxygenation (ECMO)

- Patients with refractory status epilepticus who are receiving ketamine infusion

- Proven or suspected status asthmaticus.

\section{Informed consent}

The study is conducted according to Good Clinical Practice guidelines. The study protocol as well as the informed consent have been approved by the Research Ethics Committee (REC) and Clinical Research Committee (CRC) at KFSH\&RC with Research Advisory Council (RAC) number 2191 187. Once an eligible patient is identified, study investigators start the consenting process to explain the objectives of the trial and its potential risks and benefits to the patient's surrogate decision-maker. A verbal consent from a guardian/next of kin over the phone is considered to allow randomization and initiation of timely intervention when written authorization cannot be secured in sufficient time (within $24 \mathrm{~h}$ of intubation). Verbal consent is documented in medical records to indicate the research subject's acceptance to participate in the study. A prospective written consent is obtained thereafter from the 
patient (if extubated) or the patient's guardian/next of kin once they become available.

\section{Trial interventions}

Patients will be randomized into two groups: the intervention group will receive an adjunct low-dose continuous infusion of ketamine plus the standard of care in the ICU. Ketamine will be administered over a $48-\mathrm{h}$ period at a fixed infusion rate of $2 \mu \mathrm{g} / \mathrm{kg} / \mathrm{min}(0.12 \mathrm{mg} / \mathrm{kg} / \mathrm{h})$ for the first $24 \mathrm{~h}$ followed by $1 \mu \mathrm{g} / \mathrm{kg} / \mathrm{min}(0.06 \mathrm{mg} / \mathrm{kg} / \mathrm{h})$ in the second $24 \mathrm{~h}$. The control group will receive the standard of care in the ICU, where propofol and/or fentanyl and/or midazolam will be given according to the KFSH\&RC ICU sedation and analgesia protocol as clinically appropriate. Other aspects of care in both groups, including RASS goal, SAT, SBT, mobilization, and non-pharmacological interventions to promote comfort and facilitate sleep, will be left to the discretion of the ICU attending physician. Please refer to Fig. 1: study methodology.

\section{Stopping guidelines for the intervention}

The intervention may be stopped in the following situations (see Table 1 for more details):

- The ICU team deemed that excessive sedation is persisting after holding or decreasing the other sedatives (propofol and/or fentanyl and/or midazolam) and the patient is not in target RASS.

- Adverse effects: persistent tachycardia with HR > 150 for $\geq 3 \mathrm{~h}$, persistent hypertension with SBP $>180$ for $\geq 3 \mathrm{~h}$, uncontrolled agitation (removing tubes and lines) and combative behavior.

- Patient died or goals of care changed to comfort care.

- Patient is weaned off sedation and/or extubated.

\section{Randomization}

Patients will be randomly assigned to one of two study groups in a 1:1 ratio by a computer-generated randomization
Adult patients admitted to the KFSH\&RC ICUs, intubated within the previous $24 \mathrm{~h}$, and

expected to require mechanical ventilation for more than $24 \mathrm{~h}$, and expected to be on the KFSH\&RC sedation and pain Protocol

1- Screen for eligibility and if eligible, obtain consent and then randomize

2- Randomization 1:1 ratio

Ketamine will be administered at a low dose continuous infusion fixed rate for $48 \mathrm{~h}$ :

$1^{\text {st }} 24 \mathrm{~h}: 2 \mu \mathrm{g} / \mathrm{kg} / \mathrm{min}(0.12 \mathrm{mg} / \mathrm{kg} / \mathrm{h})$

$2^{\text {nd }} 24 \mathrm{~h}: 1 \mu \mathrm{g} / \mathrm{kg} / \mathrm{min}(0.06 \mathrm{mg} / \mathrm{kg} / \mathrm{h})$

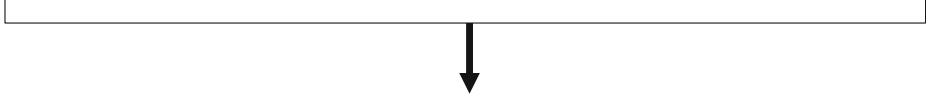

Ketamine will be discontinued if the following occurs:

- Completed the $48 \mathrm{~h}$

- If the ICU team deemed that excessive sedation is persisting after holding or decreasing the sedative and patient is not on target RASS within the first $48 \mathrm{~h}$

- Adverse effects within the first $48 \mathrm{~h}$ : persistent tachycardia $H R>150$, persistent hypertension with $\mathrm{SBP}>180$, uncontrolled agitation (removing tubes and lines) and combative behavior toward the nursing staff

- Patient died or goal of care changed to comfort care within the first $48 \mathrm{~h}$

- Patient extubated and weaned off the sedative within the first $48 \mathrm{~h}$

Fig. 1 Study methodology 
Table 1 Intervention stopping rules and protocol deviation

\begin{tabular}{|c|c|}
\hline Events & Action regarding the intervention (ketamine) \\
\hline Completed $48 \mathrm{~h}$ & $\begin{array}{l}\text { Ketamine will be discontinued (intended duration for this trial is } \\
48 \mathrm{~h} \text { ). Continuation of ketamine or other analgesics and } \\
\text { sedatives for more than } 48 \mathrm{~h} \text { will be left to the treating } \\
\text { physicians, but will not be related to the research purpose }\end{array}$ \\
\hline $\begin{array}{l}\text { Positive CAM-ICU score for delirium } \\
\text { and hallucination within the first } 48 \\
\mathrm{~h}\end{array}$ & $\begin{array}{l}\text { Ketamine will be continued, and delirium treatment (non- } \\
\text { pharmacological and antipsychotic use) will be left to the } \\
\text { treating physicians. } \\
\text { In cases of uncontrolled agitation (removal of tubes and lines } \\
\text { and combative behavior) within the first } 48 \mathrm{~h} \text {, ketamine will be } \\
\text { discontinued (refer to protocol deviation below) }\end{array}$ \\
\hline $\begin{array}{l}\text { Use of physical restraint within the } \\
\text { first } 48 \mathrm{~h}\end{array}$ & $\begin{array}{l}\text { Ketamine will be continued unless uncontrolled agitation } \\
\text { (removal of tubes and lines and combative behavior) within the } \\
\text { first } 48 \mathrm{~h} \text {, in which case ketamine will be discontinued (refer to } \\
\text { protocol deviation below) }\end{array}$ \\
\hline $\begin{array}{l}\text { Hypersalivation and frequent } \\
\text { suctioning within the first } 48 \mathrm{~h}\end{array}$ & $\begin{array}{l}\text { Ketamine will be continued and management of hypersalivation } \\
\text { will be left to the treating physicians }\end{array}$ \\
\hline
\end{tabular}

Protocol deviation (patient did not complete the intended duration of the trial (i.e., $48 \mathrm{~h}$ )

Patient or proxies withdraw consent

Patient extubated and sedation weaned off within the first $48 \mathrm{~h}$

If the ICU team believed the patient is not in target for RASS within the first $48 \mathrm{~h}$

Persistent tachycardia with $\mathrm{HR}>$ 150 for $>3 \mathrm{~h}$ within the first $48 \mathrm{~h}$

Hypertension with SBP $>180$ for

$>3 \mathrm{~h}$ within the first $48 \mathrm{~h}$

Uncontrolled agitation (pulling off tubes and lines) within the first $48 \mathrm{~h}$

Combative behavior within the first $48 \mathrm{~h}$

Patient died ${ }^{a}$ or goal of care changed to comfort care within the first $48 \mathrm{~h}$

Physician decline after randomization

Ketamine will be discontinued

When the patient is deemed to be excessively sedated after receiving ketamine and other sedatives (propofol and/or fentanyl and/or midazolam), the other sedatives will be held first (or decreased) and ketamine will be continued until the subject reaches the team's desired RASS goal. In situations where excessive sedation persisted and the patient is not yet in target RASS, then ketamine will be discontinued

When the patient is deemed to be agitated after receiving ketamine and other sedatives (propofol and/or fentanyl and/or midazolam), the other sedatives will be increased, use as needed boluses, or add dexmedetomidine. The decision to continue or discontinue ketamine infusion will be left to the discretion of the treating physicians

If the ICU treating physicians believes that ketamine is the primary causative factor, ketamine will be discontinued and patient will be followed up for $24 \mathrm{~h}$. Detailed documentation will be carried out in the medical record for adverse event, severity of event, recovery from event, group allocation, and relation to study protocol

Ketamine will be discontinued

Ketamine will be discontinued
Action regarding the study procedure

(data collection and data analysis)

Subject will be included in the data analysis

Subject will be included in the data analysis (safety outcome data)

Subject will be included in the data analysis (safety outcome data)

Subject will be included in the data analysis

All information will be removed and not included in the analysis (modified intentionto-treat principle)

Subject will be included in the data analysis

Subject will be included in the data analysis Subject will be included in the data analysis Subject will be included in the data analysis

Subject will be included in the data analysis

Subject will be included in the data analysis (safety outcome data)
Subject will be included in the data analysis

${ }^{a}$ In cases of death (either within the first $48 \mathrm{~h}$, until ICU or hospital discharge, or 28 days after randomization, whichever comes first), detailed documentation will be carried out in the medical record for the cause of death, group allocation, and relation to study protocol

list created by an independent biostatistician; no stratification will be performed. Our initial screening and eligibility assessment is done by bedside ICU nurses who are blinded to treatment assignment. To further ensure allocation concealment, access to the randomization will be restricted to a pharmacist (third party and not part of the study) to whom principal investigators refer at a distance (by telephone) to know the assigned treatment. The study investigators and study participants during the recruitment and consenting process will be blinded to the treatment assignment. Once the consenting process is complete, the principal investigators will contact the pharmacist (third party) for patient 
allocation and initiation of the trial intervention. Group allocation will be concealed until after randomization.

\section{Duration of the intervention}

The study interventions will continue for $48 \mathrm{~h}$ from the time of randomization. Patients and medical charts will be followed at baseline prior to randomization and at $24 \mathrm{~h}$ and $48 \mathrm{~h}$ post-randomization. Medical charts will also be followed to document the outcomes at 28 days, or until death, whichever comes first. Please refer to Fig. 2 for the schedule of enrollment, interventions, and assessments.

\section{Minimizing bias \\ Blinding}

The study investigators and study participants during the recruitment and consenting process will be blinded to the treatment assignment. Once the trial intervention starts, the treating team and study investigators will not be blinded to the trial intervention for practical and safety purposes (open label). The study statistician is blinded to the treatment allocation, and the study investigators will remain blinded to the results until the conclusion of the study.

\section{Minimizing contamination}

The principal investigators will ensure enrollment of patients as quickly as possible after $24 \mathrm{~h}$ post-intubation.
Patients intubated for more than $24 \mathrm{~h}$ will be excluded to eliminate early contamination or confounders.

\section{Outcomes and follow-up Primary outcome}

The primary outcome is median duration of MV: the number of calendar days from intubation date to extubation date, until ICU discharge, death, or 28 days post-randomization, whichever comes first. This outcome was chosen as a patient-centered outcome and based on the mechanistic plausibility data that showed ketamine possibly has a bronchodilatory effect and maintains respiratory drive and airway reflexes $[9,12,13]$. Because duration of MV is highly influenced by mortality, the median ventilator-free days to day 28 post-randomization will be calculated as a co-primary outcome [23]. See the statistical methods in "Data analysis."

\section{Secondary clinical outcomes}

1. Proportion and median cumulative dose of pain and sedative medications in the first $48 \mathrm{~h}$ after randomization

2. Proportion of patients started on dexmedetomidine $48 \mathrm{~h}$ after randomization

\begin{tabular}{|c|c|c|c|c|c|c|c|c|}
\hline \multirow[b]{3}{*}{ TIMEPOINT } & \multicolumn{8}{|c|}{ STUDY PERIOD } \\
\hline & \multirow{2}{*}{$\begin{array}{c}\text { Enrolment } \\
-D_{1}\end{array}$} & \multirow{2}{*}{$\frac{\text { Allocation }}{0}$} & \multicolumn{5}{|c|}{ Post-allocation } & \multirow{2}{*}{$\frac{\text { Close-out }}{\text { D28 }}$} \\
\hline & & & $D_{1}$ & $D_{2}$ & $D_{3}$ & $D_{4}$ & D.... & \\
\hline \multicolumn{9}{|l|}{ ENROLMENT: } \\
\hline \multirow{2}{*}{$\begin{array}{l}\text { Eligibility screen } \\
\text { Informed consent }\end{array}$} & $x$ & & & & & & & \\
\hline & $\mathrm{x}$ & & & & & & & \\
\hline Allocation & & $x$ & & & & & & \\
\hline \multicolumn{9}{|l|}{ INTERVENTIONS: } \\
\hline \multirow{2}{*}{$\begin{array}{r}\text { [adjunct low-dose continuous infusion of ketamine plus standard of } \\
\text { care] } \\
\text { [standard of care in the ICU] }\end{array}$} & & $x$ & $x$ & $x$ & & & & \\
\hline & & $\mathrm{x}$ & $\mathrm{x}$ & $\mathrm{x}$ & & & & \\
\hline \multicolumn{9}{|l|}{ ASSESSMENTS: } \\
\hline \multirow{2}{*}{$\begin{array}{r}\text { [baseline demographics including analgesics, sedatives, vasopressor } \\
\text { requirement, RASS, pain score, and CAM-ICU] } \\
\text { [analgesics, sedatives, vasopressor requirement, RASS, pain score, and } \\
\text { CAM-ICU, antiphsychotics, physical restraint post randomization] }\end{array}$} & $x$ & & & & & & & \\
\hline & & & $x$ & $x$ & & & & \\
\hline $\begin{array}{l}\text { [Duration of Mechanical } \\
\text { Ventilation, tracheostomy, unplanned extubation, and re-intubation] }\end{array}$ & & & $x$ & $x$ & $x$ & $x$ & $x$ & $\mathrm{x}$ \\
\hline \multirow{2}{*}{ [Length of Stay] } & & & $x$ & $x$ & $\mathrm{x}$ & $x$ & $x$ & $x$ \\
\hline & & & $x$ & $x$ & $x$ & $x$ & $x$ & $\mathrm{x}$ \\
\hline
\end{tabular}

Fig. 2 Schedule of enrollment, interventions, and assessments for Analgo-sedative adjuncT keTAmine Infusion iN Mechanically vENTilated ICU patients (ATTAINMENT trial) 
3. Proportion of patients achieving the RASS goal and pain score goal within the first $48 \mathrm{~h}$ after randomization

4. Proportion and median vasopressor requirements in the first $48 \mathrm{~h}$ after randomization

5. Median change in mean arterial pressure (MAP) and $\mathrm{HR}$ in the first $48 \mathrm{~h}$ after randomization

6. ICU and hospital LOS: number of calendar days (median, IQR) from randomization to discharge date from the ICU or hospital

7. Proportion of tracheostomy, unplanned extubation (self-extubation), and re-intubation within 28 days post-randomization

8. Proportion of patients starting on antipsychotics and positive CAM-ICU score to assess the incidence of delirium $48 \mathrm{~h}$ after randomization. The presence of delirium will also be confirmed through a psychiatrist consultation

9. Proportion of physical restraint $48 \mathrm{~h}$ after randomization

10. Proportion of patients with frequent suctioning in the first $48 \mathrm{~h}$ after randomization (defined as interval between suctioning episodes $2 \mathrm{~h}$ or less)

11. Mortality rate at the time of hospital discharge or 28 days after randomization, whichever comes first.

\section{Secondary feasibility outcomes:}

1. Proportion of screened patients

2. Proportion of eligible patients enrolled

3. Enrollment rate (i.e., number of enrollments per month)

4. Protocol compliance.

\section{Data management}

Data will be collected in the KFSH\&RC Research Electronic Data Capture (REDCap) platform. Each subject will be given a unique subject ID number (database numbers and all identifiers will be removed). A subject ID key will be used to match the subjects' Medical Record Numbers and will be kept in a password-protected file that is accessible to the principal investigators. Access to the RedCap data will be limited to the principal investigators and co-investigators involved in data collection only. Access to REDCap requires authentication (username and password) for secure maintenance of the data. All investigators are KFSH\&RC employees and have access to the electronic medical record (Power Chart). All collected information will be stored in a secure manner, and all patient data will be kept confidential. To ensure consistency in data collection, training sessions will be held by the principal investigators for all research co-investigators involved in data collection prior to study commencement. Additionally, the principal investigators will conduct educational sessions for ICU physicians and ICU nurses, which will include the study protocol, and periodic follow-up educational sessions to provide feedback and ensure optimal compliance with the study protocol. There will be periodic internal audits of data entry accuracy and compliance by the principal investigators. This will allow us to identify any protocol deviations and provide an opportunity for feedback to the co-investigators involved in data entry. Range edits and value checks will be incorporated into the REDCap software to minimize the potential for data entry errors. Moreover, printed copies of de-identified Case Report Forms will be submitted to the RAC at the KFSH\&RC any time upon committee request and will be reviewed upon the receipt of the progress report by 4 June 2020 (the date specified originally upon IRB approval of the study protocol).

The following data will be collected: age, gender, weight, mode of MV at baseline, percentage of renal replacement therapy at baseline, lactate level at baseline, and severity of illness as estimated by Sequential Organ Failure Assessment (SOFA) score and Acute Physiology and Chronic Health Evaluation (APACHE II) score, with higher scores indicating higher severity of illness [24]. Moreover, we will collect ICU type, baseline analgesics, sedatives, vasopressor requirements, and PRE-DELIRIC Delirium Risk Score, which is a delirium prediction model specifically designed for adult critical care patients $24 \mathrm{~h}$ after ICU admission. This model will be used to predict the factors that may influence delirium risk prior to randomization [25]. We will also collect RASS, pain, and CAM-ICU scores at baseline and at 24 and 48 $\mathrm{h}$ post-randomization. The RASS is a scale used to assess the depth of sedation on a scale of -5 to +4 , with a negative value indicating deeper sedation and positive values indicating increased agitation [1]. The CAM-ICU is a valid and reliable delirium assessment tool. Patients with a RASS score of -3 or lower will be excluded from CAM-ICU assessment, as they cannot participate in the exam [26]. We will calculate the modified Clinical Pulmonary Infection Score (CPIS) to differentiate secretions caused by patients' underlying lung pathology (ventilator-associated pneumonia [VAP]) vs ketamine-associated hypersalivation [27]. We will also record the proportion of eligible participants enrolled, rates of recruitment, protocol deviations, and AEs.

\section{Data analysis}

The sample size calculation associated with the specified (required) number of patients to be recruited is based on the study by Buchheit et al. [9]. In their study, the median time from initiation of ketamine to extubation was 1.44 days (IQR 0.58-2.66). As time from initiation of ketamine to extubation is bounded below by zero, and 
assuming that the distribution is skewed toward larger times, a lognormal distribution was assumed to be appropriate for modeling such times.

We additionally assumed that the distribution of times from intubation to extubation for patients who are not treated with ketamine is lognormal, with median 2.44 and IQR from 1.60 to 4.00 .

The hypothesis of interest is $\mathrm{H} 0: \mathrm{m} 1=\mathrm{m} 2$ vs Ha: $\mathrm{m} 1 \neq \mathrm{m} 2$, where $\mathrm{m} 1$ is the median time for those treated with ketamine, and $\mathrm{m} 2$ is the median time for those not treated with ketamine. Here the times are distributed with lognormal distributions, and the respective IQRs are $0.58-2.66$ for ketamine-treated patients and 1.604.00 for those not treated with ketamine. A simulation analysis was carried out with 35 simulated patient times under each of the above two distributional scenarios, i.e., 35 intubation to extubation times under a lognormal of 1.44 and $0.58-2.66$ (median and IQR) and 35 intubation to extubation times under a lognormal of 2.44 and 1.60 4.00 (median and IQR). This was followed by calculation of the level of significance by the Wilcoxon rank sum test (i.e., the associated $P$ value). By repeating this simulation 10,000 times, $80.24 \%$ of the simulations had a $P$ value less than 0.05 . This shows that the power of a design with 35 ketamine-treated and 35 non-ketaminetreated patients should (with at least $80 \%$ probability) demonstrate that the time from intubation to extubation is one day less for those treated with ketamine. It is recognized that non-compliance and dropouts may occur. Hence, the study has been designed to recruit 40 ketamine-treated and 40 untreated subjects for analyses (i.e., total sample size 80 ). The median ventilator-free days will be calculated as calendar days with no ventilator support to day 28 post-randomization. Participants who die before day 28 are assigned zero free days. Data will be analyzed using the modified intention-to-treat principle and will comprise data from all patients who undergo randomization, with the exception of those who withdraw consent, have an unknown primary outcome, or are identified as ineligible after randomization. The Shapiro-Wilk test for normality will be used to assess the distribution of all outcome variables. Chi-square and $t$ tests (or Wilcoxon rank sum) will be used to compare categorical data and continuous data, respectively. All data will be presented as median and IQR, if not normally distributed (or count and percentages, if categorical). Univariate and multivariate regression analyses will be used to identify risk factors and predictors for delirium. Statistically significant factors in the univariate analysis $(\leq 0.05)$ will be included in the multivariate analysis. Adjustments for the analysis will be accounted for with the Bonferroni technique. A prespecified sub-group analysis of the primary outcome will be conducted on the following variables:
- Age $>60$ vs age $<60$

- SOFA score $>10$ vs $<10$

- APACHE II $>20$ vs $<20$

- Ratio of the partial pressure of arterial oxygen to the fraction of inspired oxygen (PF ratio $>150$ vs PF ratio $<150$ )

- Surgical vs medical admission.

We will strive to obtain full data on every patient to allow an intention-to-treat analysis. If there is missing information as patients withdraw from the study before completion of the follow-up period, it will be handled in the normal fashion of survival analysis (censored observation). Imputation (based on regression model) will be considered in case of incomplete information about key covariates. Sensitivity analyses with such excluded patients will be conducted and compared with an imputed values model. Each of the analyses will be redone to test each hypothesis and verify the robustness of the conclusion. Statistical analyses will be performed using SAS/ JMP, v.14.1 (SAS Institute, Cary, NC, USA). The study statistician is blinded to the treatment allocation, and study investigators will remain blinded to the results until the conclusion of the study.

\section{Trial administration and Safety Monitoring Committee}

The principal investigators will meet weekly to perform periodic internal audits of data accuracy, review enrollment rates, and oversee and coordinate the study in general. This will allow them to identify any protocol deviations and provide an opportunity for feedback to the other co-investigators.

An independent RAC at the KFSH\&RC will serve as a Safety Monitoring Committee which includes faculty with expertise in various disciplines engaged in human subjects' research from the hospital and research center, and also community members. Consultants with special expertise might be invited to assist from time to time with complex issues. The committee will undertake periodic reviews at the discretion of the Chair, and an expedited review is done for all serious unexpected adverse events (SUAEs), including death. The committee has the authority to suspend or halt recruitment if necessary. Refer to Supplementary File 2 for the form used to report SUAEs and death by the study investigators within $48 \mathrm{~h}$ of occurrence. All death cases reviewed so far have been due to the underlying disease, with participation in the trial not being a contributing factor. Any clinically significant worsening in a study participant's condition based on clinical judgement compared to the baseline status at the time of randomization will be recorded as an $\mathrm{AE}$ in our progress report to be sent to the RAC by 4 June 2020 (the date specified originally upon IRB approval of the study protocol). This is applied whether or 
not the $\mathrm{AE}$ is considered to be related to the study treatment. In addition, this study is registered at the Saudi Food and Drug Authority (FDA), which provides independent input regarding the safety of interventions. Since this is an investigator-initiated, single-center, pilot, feasibility trial, periodic reviews are basically focused on monitoring safety. No formal interim analysis of efficacy will be undertaken due to possible small numbers that might preclude determination of a statistically significant difference in outcomes between the arms. No stopping rules or external independent Data Safety Monitoring Committee (DSMC) are specified. We believe the administration of sedative agents is standard of practice in the ICU to minimize a patient's discomfort while on MV (see Supplementary Figure 1: KFSH\&RC new sedation protocol for adult ICUs). Hence, the expected adverse effects will not exceed what is encountered during daily practice (e.g., benzodiazepine-associated delirium, opioid-induced constipation, hemodynamic instability associated with propofol and dexmedetomidine, ketamine-associated sympathetic stimulation " tachycardia and increase in blood pressure," and possible delirium). Nonetheless, an external and independent DSMC will be considered moving forward to a multisite RCT.

\section{Ancillary and post-trial care}

As detailed in the Patient Information and Consent Form, any injury or complication occurring as a result of trial participation is to be reported to the study team, who will arrange all necessary medical treatment.

\section{Trial disseminationThe trial registration and dissemination information is as follows:}

1. The trial was registered at ClinicalTrials.gov: Identifier NCT04075006 (registered on 30 August 2019), Saudi Food and Drug Authority: SCTR \#19063002 (registered on 27 August 2019), and current controlled trials: ISRCTN14730035 (registered on 3 February 2020).

2. Trial results are to be presented at relevant scientific meetings and published in peer-reviewed journals.

3. The role of adjunct ketamine infusion as analgosedation is presented to the Saudi Critical Care Trials Group.

4. The trial will be publicized by ISRCTN via social media, and a trial blog will be available at the BMC website.

\section{Discussion}

To the best of our knowledge, our pilot study is the first RCT that compares adjunct low-dose ketamine infusion to standard of care alone in critically ill patients. It is conducted in a mixed ICU cohort (medical, surgical, transplant, and oncology ICU settings), focused on patient-centered outcomes as a primary outcome (duration of MV), and addresses the fact that most patients in the ICU are sedated with a combination of drugs. Randomization, blinded study participants and study statistician, and adherence to the modified intention-to-treat principle will limit potential sources of bias. Another strength of this pilot study is the narrow randomization window (within $24 \mathrm{~h}$ post-intubation), which was chosen based on prior literature that showed early initiation of an intervention increases the ability of the intervention to influence the outcome, be more informative for clinicians, and have a greater power to detect an effect on important outcomes, such as duration of mechanical ventilation and long-term outcomes [16-18]. Moreover, we elected to record the vasopressor requirements, cumulative sedatives and analgesics, number of patients within RASS and pain score goals, and delirium incidence $48 \mathrm{~h}$ post-randomization to avoid the presence of confounders if measured $>48 \mathrm{~h}$ after ketamine infusion, similar to the study by Groetzinger et al. [11].

A concern was raised about the under-dosing of ketamine compared to ICU ketamine studies. Various dosing regimens of ketamine continuous infusion for sedation are described in the literature. A recent systematic review described the existing data regarding ketamine dosing for adjunct sedation in small cohorts of primarily neurologically injured patients [12]. The included studies describe dosing regimens up to $103.3 \mu \mathrm{g} / \mathrm{kg} / \mathrm{min}(6.2$ $\mathrm{mg} / \mathrm{kg} / \mathrm{h}$ ), which is substantially higher than the doses prescribed in our cohort. As most patients in the review had a neurological injury, sedatives were administered to maintain deep sedation, and often with background benzodiazepine infusion or even barbiturate anesthesia [12]. On the other hand, Groetzinger and colleagues used continuous infusion ketamine for adjunct sedation in a population of mechanically ventilated, critically ill adults targeting light sedation; ketamine was infused at a median starting dose of $1.6-4.2 \mu \mathrm{g} / \mathrm{kg} / \mathrm{min}(0.1-0.25$ $\mathrm{mg} / \mathrm{kg} / \mathrm{h}$ ) for a median of 2.8 days. The maximum doses of ketamine in individual patients experiencing adverse drug reactions (such as tachyarrhythmia) ranged from $2.08-20 \mu \mathrm{g} / \mathrm{kg} / \mathrm{min}$ (0.125 to $1.2 \mathrm{mg} / \mathrm{kg} / \mathrm{h}$ ), necessitating discontinuation of the infusion [11].

We aimed to describe our experience using ketamine as an adjunct low-dose sedative in an era that emphasizes light sedation in a complex, mechanically ventilated, critically ill population, specifically patients with medical illnesses, or following complicated surgical procedures. Therefore, we chose in our pilot study the dosing regimen based on the RCT cited by the 2018 PADIS guideline, which is comparable to the dosing regimen described in the study of Groetzinger et al. We believe this represents the safest dose as an adjunct analgo- 
sedative agent to decrease the risk of side effects, i.e., delirium, hallucinations, and tachycardia, through its sympathetic stimulation $[1,3,11]$.

Limitations of our pilot study include the open-label design, as it has a process of multiple interventions related to the pain and sedation protocol. Therefore, the ICU treating team and the study investigators will know to which arm the study participants are randomized. Moreover, we will not collect data on other pain medications such as morphine and hydromorphone, as those medications are rarely used, per the KFSH\&RC adult sedation protocol, compared to fentanyl. Another limitation is exclusion of patients in status asthmaticus or status epilepticus and patients placed on ECMO, as the dosing regimen of ketamine in those conditions is different than the regimen we used herein as an adjunct analgo-sedative agent. This may limit the external validity of this trial, although the population described in our cohort is relevant to many critically ill patients. Since we were interested in describing ketamine infusions as part of a light sedation strategy, we have excluded patients for whom the RASS goal is -5 , such as those receiving continuous infusion neuromuscular blockade. Additionally, the safety profile described in our cohort is not generalizable to higher doses of ketamine used to achieve deep sedation.

In conclusion, the findings of this pilot trial will contribute to a better understanding of adjunct low-dose ketamine infusion as an analgo-sedative agent and test the feasibility for a larger multicenter, randomized, double-blind, placebo-controlled trial with an adequate power to determine the effect of ketamine infusion as an analgo-sedative agent on clinical outcomes- mirroring other major sedation-related RCTs [18, 23, 28-30]. Future trials addressing cardiac assessment and hemodynamic metrics in a more protocolized way would be a great addition. For example, studies could assess metrics such as measurement of cardiac index (CI), stroke volume (SV), pulse pressure variation (PPV), and stroke volume variation (SVV) estimated by arterial pulse pressure waveform analysis (e.g., with the Vigileo monitor) at baseline (prerandomization) and at 24 and $48 \mathrm{~h}$ afterward (post-randomization), considering other potential confounders and adjunct interventions to evaluate whether these findings are direct consequences of ketamine or independent changes related to the severity of critical illness and the cumulative effect of adjunct interventions (i.e., antimicrobials, steroids, fluid administration, and blood products). Lastly, it would be optimal to assess some patient-reported outcomes for those who developed an emergence reaction or delirium. The active engagement of patients and family members has been highlighted recently in the ICU literature, and this would be a great addition to consider in a future multicenter trial through completing an ICU diary or a survey 28 days post-ICU discharge among ICU survivors [31].

\section{Trial status}

As of 10 October 2019, a total of 16 patients have been enrolled. We expect to complete the recruitment by 31 December 2020. The trial was first approved on 13 July 2019 (Protocol V1) and opened to recruitment on 1 September 2019 (Protocol V1). The protocol was amended on 3 September 2019 (Protocol V2) requesting initial waiver of consent, as we faced difficulty with the patient enrollment and consenting prior to randomization due to inability to reach the legal surrogate (not answering the phone for verbal consent) or the emotional factor with the legal surrogate, especially during the first $24 \mathrm{~h}$ postintubation and ICU admission. However, our Research Ethics Committee mandated the informed consent prior to randomization. Another protocol amendment (Protocol V3) on 24 February 2020 reflected the clarifications that were made in the revised version.

\section{Supplementary information}

Supplementary information accompanies this paper at https://doi.org/10. 1186/s13063-020-4216-4.

Additional file 1: Supplementary Table 1. Previous ketamine trials in the ICU setting. Supplementary Figure 1. KFSH\&RC new sedation protocol for adult intensive care units. Supplementary File 1. Standard Protocol Items: Recommendations for Interventional Trials (SPIRIT) checklist. Supplementary Table 2. Adjunct low-dose ketamine infusion vs standard of care in mechanically ventilated critically ill patients at a tertiary Saudi hospital (ATTAINMENT trial) SPIRIT protocol summary. Supplementary File 2. Serious Unexpected Adverse Event (SUAE) Report Form.

\section{Abbreviations}

AE: Adverse event; APACHE II: Acute Physiology and Chronic Health Evaluation II; Cl: Cardiac index; CRC: Clinical Research Committee; CAMICU: Confusion Assessment Method for the ICU; DSMC: Data Safety Monitoring Committee; DNR: Do Not Resuscitate; ECMO: Extracorporeal membrane oxygenation; HR: Heart rate; ICU: Intensive care unit;

IQR: Interquartile range; IRB: Institutional Review Board; KFSH\&RC: King Faisal Specialist Hospital and Research Center; LOS: Length of stay; MAP: Mean arterial pressure; MV: Mechanical ventilation; CPIS: Modified Clinical Pulmonary Infection Score; ORA: Office of Research Affairs; PADIS: Pain, Agitation/sedation, Delirium, Immobility, and Sleep disruption; $\mathrm{RCT}$ : Randomized controlled trial; PF ratio: Ratio of the partial pressure of arterial oxygen to the fraction of inspired oxygen; REC: Research Ethics Committee; REDCap: Research Electronic Data Capture; RASS: Richmond Agitation-Sedation Scale; SOFA: Sequential Organ Failure Assessment; SUAE: Serious unexpected adverse event; SAT: Spontaneous awakening trial; SBT: Spontaneous breathing trial; SV: Stroke volume; SW: Stroke volume variation; SBP: Systolic blood pressure; VAP: Ventilator-associated pneumonia

\section{Acknowledgements}

We thank the ICU physicians, ICU nurses, ICU nurses' clinical educators, ICU satellite pharmacists, and the Pharmacy Automation Team at KFSH\&RC for their support to this study. We are thankful to the Saudi Critical Care Trials Group for providing feedback for the study proposal. We also thank the research staff, and the participants and their families. Without their collective generosity, this trial would not have been possible. 


\section{Authors' contributions}

$\mathrm{MB}$ and MA conceived and designed the study and the analytical plan, drafted the manuscript, critically revised the manuscript for important intellectual content, and registered the trial at Saudi FDA, ClinicalTrials.gov, and ISRCTN. Dr. MB and Dr. MA contributed equally as first authors, have full access to all of the data in the study, and take responsibility for the integrity of the data, study supervision, accuracy of the data analysis, and approval of the final version of the study protocol to be published. KM acquired, analyzed, and interpreted the data, critically revised the manuscript for important intellectual content, and approved the final version to be published. MH acquired, analyzed, and interpreted the data, critically revised the manuscript for important intellectual content, and approved the final version to be published. RA, MR, KA, and MS acquired, analyzed, and interpreted the data and approved the final version to be published. ED participated in sample size calculation, analysis or interpretation of data, submission of the documents to the Saudi FDA, and approval of the final version to be published. We confirmed that the authorship followed the uniform requirements for manuscripts submitted to biomedical journals. All authors read and approved the final manuscript.

\section{Authors' information}

MB: MD, FRCSC, FACS, Medical Director, Surgical Critical Care Unit, Consultant Trauma and Acute Care Surgery, Critical Care Medicine, Department of Critical Care Medicine (MBC 94), King Faisal Specialist Hospital \& Research Centre, P.O Box 3354, Riyadh 11211, Tel: +966-11-216-2919, Ext: 24731. MA: PharmD, BCPS, BCCCP, Critical Care Pharmacy Specialist, Medical and Surgical ICU, Adjunct Assistant Professor- Alfaisal University, Pharmaceutical Care Division (MBC 11), King Faisal Specialist Hospital \& Research Center PO Box 3354, Riyadh, 11211, Tel: +966114647272 Ext 70836. KM: MD, FCCP, MRCP (UK), Chairman and Consultant Intensivist, Department of Critical Care Medicine (MBC 94), King Faisal Specialist Hospital \& Research Centre, P.O Box 3354, Riyadh 11211, Tel: +966-11-442-4731. MH: MD, FACP, FCCP, Consultant Intensivist, Department of Critical Care Medicine (MBC 94), King Faisal Specialist Hospital \& Research Centre, P.OBox 3354, Riyadh 11211, Tel: +96611-464-7272 Ext 37165. RA: PharmD, BCPS, BCCCP, Critical Care Pharmacy Specialist, Medical and Surgical ICU, Pharmaceutical Care Division (MBC 11), King Faisal Specialist Hospital \& Research Center, PO Box 3354, Riyadh, 11211. MS: MD, Assistant consultant Department of Critical Care Medicine (MBC 94), King Faisal Specialist Hospital \& Research Centre, P.O Box 3354, Riyadh 11211, Tel: +966-11-464-7272 Ext 37165. KA: MD, Assistant consultant, Department of Critical Care Medicine (MBC 94), King Faisal Specialist Hospital \& Research Centre, P.O Box 3354, Riyadh 11211, Tel: +966-11-464-7272 Ext 37165. MR: MD, Assistant consultant, Department of Critical Care Medicine (MBC 94), King Faisal Specialist Hospital \& Research Centre, P.O Box 3354, Riyadh 11211, Tel: +966-11-464-7272 Ext 37165. ED: Chairman, Department of Biostatistics, Epidemiology and Scientific Computing, King Faisal Specialist Hospital \& Research Centre, P.O Box 3354, Riyadh 11211, Tel: +966-11-464-7272 Ext 32504

\section{Funding}

This trial is investigator-initiated, and all study authors are employees at King Faisal Specialist Hospital and Research Center (KFSH\&RC), which has not provided any research grant for this particular project. All authors are expected to volunteer their time and use local resources to conduct the study. The study drug ketamine is provided to the KFSH\&RC Pharmaceutical Care Division through Hikma Pharmaceuticals, which has no role in the design or conduct of the trial, analysis of the data, or writing or review of the manuscript. Email: ORA@kfshrc.edu.sa.

\section{Availability of data and materials}

All data in the study protocol are included in this published article and its supplementary information files.

\section{Ethics approval and consent to participate}

The study is conducted according to Good Clinical Practice guidelines. The study protocol and informed consent were approved by the Research Ethics Committee and Clinical Research Committee at KFSH\&RC with Research Advisory Council number 2191 187. Once an eligible patient is identified, study investigators start the consenting process to explain the objectives of the trial and its potential risks and benefits to the patient's surrogate decision-maker. A verbal consent from a guardian/next of kin over the phone is considered to allow randomization and initiation of timely intervention when written authorization cannot be secured in sufficient time (within $24 \mathrm{~h}$ of intubation). Verbal consent is documented in medical records to indicate the research subject's acceptance to participate in the study. A prospective written consent is obtained thereafter from the patient (if extubated) or the patient's guardian/next of kin once they become available.

\section{Consent for publication}

Not applicable.

\section{Competing interests}

The authors declare that they have no competing interests.

\section{Author details}

'Department of Critical Care Medicine (MBC 94), King Faisal Specialist Hospital and Research Center, P.O Box 3354, Riyadh 11211, Saudi Arabia. ${ }^{2}$ Pharmaceutical Care Division (MBC 11), King Faisal Specialist Hospital and Research Center, P.O Box 3354, Riyadh 11211, Saudi Arabia. ${ }^{3}$ Department of Biostatistics, Epidemiology and Scientific Computing, King Faisal Specialist Hospital and Research Center, P.O Box 3354, Riyadh 11211, Saudi Arabia.

Received: 28 November 2019 Accepted: 28 February 2020

Published online: 20 March 2020

\section{References}

1. Devlin JW, Skrobik Y, Gelinas C, Needham DM, Slooter AJC, Pandharipande PP, et al. Clinical Practice Guidelines for the Prevention and Management of Pain, Agitation/Sedation, Delirium, Immobility, and Sleep Disruption in Adult Patients in the ICU. Crit Care Med. 2018;46:e825-73.

2. Whitman CB, Rhodes HM, Tellor BR, Hampton NB. Continuous infusion ketamine for adjunctive sedation in medical intensive care unit patients: a case series. Enliven: J Anesthesiol Crit Care Med. 2015;2(5):1-5.

3. Guillou N, Tanguy M, Seguin P, Branger B, Campion JP, Mallédant Y. The effects of small-dose ketamine on morphine consumption in surgical intensive care unit patients after major abdominal surgery. Anesth Analg. 2003;97:843-7.

4. Moitra VK, Patel MK, Darrah D, Moitra A, Wunsch H. Low-dose ketamine in chronic critical illness. J Intensive Care Med. 2015;31(3):1-5.

5. Garber PM, Droege CA, Carter KE, Harger NJ, Mueller EW. Continuous infusion ketamine for adjunctive analgosedation in mechanically ventilated, critically ill patients. Pharmacotherapy. 2019;39(3):288-96.

6. Pruskowski KA, Harbourt K, Pajoumand M, Chui SJ, Reynolds HN. Impact of ketamine use on adjunctive analgesic and sedative medications in critically ill trauma patients. Pharmacotherapy. 2017;37(12):1537-44.

7. Bourgoin A, Albanese J, Wereszczynski N, Charbit M, Vialet R, Martin C. Safety of sedation with ketamine in severe head injury patients: comparison with sufentanil. Crit Care Med. 2003;31(3):711-7.

8. Shurtleff $\mathrm{V}$, Radosevich JJ, Patanwala AE. Comparison of ketamine- versus nonketamine-based sedation on delirium and coma in the intensive care unit. J Intensive Care Med 2018. doi: https://doi.org/10.1177/ 0885066618767619.

9. Buchheit JL, Yeh DD, Eikermann M, Lin H. Impact of low-dose ketamine on the usage of continuous opioid infusion for the treatment of pain in adult mechanically ventilated patients in surgical intensive care units. J Intensive Care Med. 2019:34(8):646-51.

10. Perbet S, Verdonk F, Godet T, Jabaudon M, Chartier C, Cayot S, et al. Low doses of ketamine reduce delirium but not opiate consumption in mechanically ventilated and sedated ICU patients: a randomized doubleblind control trial. Anaesth Crit Care Pain Med. 2018:37(6):589-95.

11. Groetzinger LM, Rivosecchi RM, Bain W, Bahr M, Chin K, McVerry BJ, et al. Ketamine infusion for adjunct sedation in mechanically ventilated adults. Pharmacotherapy. 2018;38(2):181-8.

12. Patanwala AE, Martin JR, Erstad BL. Ketamine for analgosedation in the intensive care unit: a systematic review. J Intensive Care Med. 2017;32(6): 387-95.

13. Wieruszewski PM, Leung JG, Nelson S. Ketamine use in the intensive care unit. AACN Adv Crit Care. 2018;29(2):101-6. https://doi.org/10.4037/ aacnacc2018448.

14. Radvansky BM, Shah K, Parikh A, Sifonios AN, Le V, Eloy JD. Role of ketamine in acute postoperative pain management: a narrative review. Biomed Res Int. 2015;2015:749837. 
15. Cohen SP, Bhatia A, Buvanendran A, Schwenk ES, et al. Consensus guidelines on the use of intravenous ketamine infusions for chronic pain from the American Society of Regional Anesthesia and Pain Medicine, the American Academy of Pain Medicine, and the American Society of Anesthesiologists. Reg Anesth Pain Med. 2018;43(5):521-46.

16. Shehabi Y, Bellomo R, Reade MC, Bailey M, Bass F, Howe B, et al. Early goaldirected sedation versus standard sedation in mechanically ventilated critically ill patients: a pilot study. Crit Care Med. 2013;41(8):1983-91.

17. Shehabi Y, Forbes AB, Arabi Y, Bass F, Bellomo R, Kadiman S, et al. The SPICE III study protocol and analysis plan: a randomized trial of early goal-directed sedation compared with standard care in mechanically ventilated patients. Crit Care Resusc. 2017;19(4):318-26.

18. Shehabi Y, Howe BD, Bellomo, Arabi YM, Bailey M, Bass FE, et al. Early sedation with dexmedetomidine in critically ill patients (SPICE III). N Engl J Med. 2019;380(26):2506-17.

19. Golding CL, Miller JL, Gessouroun MR, et al. Ketamine continuous infusions in critically ill infants and children. Ann Pharmacother. 2016;50(3):234-41.

20. Strayer RJ, Nelson LS. Adverse events associated with ketamine for procedural sedation in adults. Am J Emerg Med. 2008;26:985-1028.

21. ClinicalTrials.gov. Comparing clinical outcomes between ketaminemidazolam and morphine-midazolam for continuous sedation in ICU patients. (KeMiMof). https://clinicaltrials.gov/ct2/show/NCT03407404?cond= ketamine+sedation+in+icu\&rank=2. Accessed 25 Sep 2019.

22. Manasco AT, Stephens RJ, Yaeger LH, Roberts BW, Fuller BM. Ketamine sedation in mechanically ventilated patients: a systematic review and metaanalysis. J Crit Care. 2019:56:80-8.

23. Yehya N, Harhay MO, Curley MAQ, Schoenfeld DA, Reeder RW. Reappraisal of ventilator-free days in critical care research. Am J Respir Crit Care Med. 2019;200(7):828-36.

24. Singer M, Deutschman CS, Seymour CW, Shankar-Hari M, Annane D, Bauer $\mathrm{M}$, et al. The third international consensus definitions for sepsis and septic shock (sepsis-3). JAMA. 2016;315(8):801-10

25. Van den Boogaard M, Pickkers P, Slooter AJ, Kuiper MA, Spronk PE, van der Voort PH, et al. Development and validation of PRE-DELIRIC (PREdiction of DELIRium in ICu patients) delirium prediction model for intensive care patients: observational multicentre study. BMJ. 2012;344:e420.

26. Aljuaid MH, Deeb AM, Dbsawy M, Alsayegh D, Alotaibi M, Arabi YM, et al. Psychometric properties of the Arabic version of the confusion assessment method for the intensive care unit (CAM-ICU). BMC Psychiatry. 2018;18:91 Arabic version of CAM-ICU is available at https:/www.icudelirium.org/ medical-professionals/downloads/resource-language-translations. Accessed 25 Sep 2019.

27. Fartoukh M, Maitre B, Honoré S, Cerf C, Zahar JR, Brun-Buisson C. Diagnosing pneumonia during mechanical ventilation the clinical pulmonary infection score revisited. Am J Respir Crit Care Med. 2003;168(2): 173-9.

28. Jakob SM, Ruokonen E, Grounds RM, Sarapohja T, Garratt C, Pocock SJ. at al. Dexmedetomidine vs midazolam or propofol for sedation during prolonged mechanical ventilation: two randomized controlled trials (PRODEX-MIDEX). JAMA. 2012;307(11):1151-60.

29. Pandharipande PP, Pun BT, Herr DL, Maze M, Girard TD, Miller RR, et al. Effect of sedation with dexmedetomidine vs lorazepam on acute brain dysfunction in mechanically ventilated patients: the MENDS randomized controlled trial. JAMA. 2007;298(22):2644-53.

30. Riker RR, Shehabi Y, Bokesch PM, Ceraso D, Wisemandle W, Koura F, et al. Dexmedetomidine vs midazolam for sedation of critically ill patients: a randomized trial (SEDCOM). JAMA. 2009:301(5):489-99.

31. Geense WW, van den Boogaard M, van der Hoeven JG, et al. Nonpharmacologic interventions to prevent or mitigate adverse long-term outcomes among ICU survivors: a systematic review and meta-analysis. Crit Care Med. 2019:47:1607-18.

\section{Publisher's Note}

Springer Nature remains neutral with regard to jurisdictional claims in published maps and institutional affiliations.

\section{Ready to submit your research? Choose BMC and benefit from:}

- fast, convenient online submission

- thorough peer review by experienced researchers in your field

- rapid publication on acceptance

- support for research data, including large and complex data types

- gold Open Access which fosters wider collaboration and increased citations

- maximum visibility for your research: over $100 \mathrm{M}$ website views per year

At BMC, research is always in progress.

Learn more biomedcentral.com/submissions 